\title{
Author Index to Volume 27
}

\author{
Aarnoudse JG, 442 \\ Abe Y, 11 \\ Abumrad N, 519 \\ Alfthan $\mathrm{H}, 215$ \\ Al-Mahroos FT, 519 \\ Alm GV, 7 \\ Alpan G, 133 \\ Alston JT, 122 \\ Amir J, 358 \\ Anderson GJ, 89 \\ Andersson S, 137 \\ Antila KJ, 383 \\ Antonozzi I, 297 \\ Arvat E, 405 \\ Asayama K, 472 \\ Avery ME, S20 \\ Avison MJ, 566 \\ Axelsson I, 4 \\ Aziz SM, 344
}

Bachmann C, 297, 566

Balaraman V, 392

Balfe A, 304

Balloch A, 365

Bancalari E, 327

Bandini LG, 198

Barker PM, 588

Barrington $\mathrm{KJ}, 118$

Bassett NS, 401

Bauer K, 256

Bell JM, 181

Benet LZ, 282

Benso L, 405

Bergstrom WH, 571

Berman I, 41

Bernfield M, S21

Bezemer AC, 514

Bhan AK, 239

Binoux M, 488

Blumberg DA, 353

Bonnefont J-P, 497

Boquist L, 41

Bowdy BB, 344

Bowdy BD, 349

Boynton BR, 64

Breier BH, 401

Buehlamnn U, 508

Bulus N, 519

Burnell EE, 52

Burns JC, 109

Buts J-P, 161

Butzner JD, 245

Cabrol S, 488

Cairo MS, 612

Callahan DJ, 186

Calvert SA, 552

Carey RM, 396

Castillo RO, 492

Cederbaum SD, 297

Cederblad B, 7

Cella SG, 405

Cepanec C, 497

Cerone R, 297

Chalmers RA, 413

Chamoles N, 297

Chanock RM, S39

Charlton VE, 274

Chen WW, 304
Cherry JD, 353

Chevalier RL, 396

Childs B, S8

Christensen MA, 450

Christensen RD, 583

Christenson PD, 353

Clifford PS, 148

Clyman RI, 133

Colin AW, 204

Collins Jr JW, 56

Colombo JP, 297, 566

Connor WE, 89

Cook WR, 293

Coon RL, 148

Coonrod JD, 344

Corda R, 405

Corliss JD, 89

Cornaglia-Ferraris $\mathrm{P}, 1$

Crinella FM, 181

Daniels M, 337

Davidson D, 128

Davidson LA, 176

Davis JM, 460

De Deyn PP, 297

De Graaf SSN, 256

De Keyser N, 161

del Pizzo R, 85

Demaugre F, 497

Denny Jr FW, S49

Dickerson B, 460

Dietz WH, 198

Di Martino D, 1

Dobashi K, 472

Döhlemann C, 278

Donnell GN, 117

Donti $\mathrm{E}, 1$

Dunkel L, 211, 215

Easa D, 392

Eldemerdash A, 128

Elias W, 137

Ellis L, 32

Engle MJ, 186

Engström KG, 220

Eshel GM, 264

Farrar S, 476

Finer NN, 118

Finley SL, 56

Fisher JE, 153

Fjeld CJ, 98

Fleer A, 514

Foster P, 617

Frantz ID, III, 64

Freihorst J, 153

Frerman FE, 311

Freundt-Thurne J, 98

Friedman WF, 144

Friis-Hansen B, 445

Fuerer E, 508

Fukuda Y, 260

Fyhrquist F, 137

Gadjusek DC, S59

Gall DG, 245

Gardiner RM, 436

Garza C, 252
Gatti R, 297

Geelen SPM, 514

Gerards LJ, 514

Ghigo E, 405

Ghishan FK, 519

Gibson Jr RL, 466

Gillespie MN, 344, 349

Girard F, 488

Gluckman PD, 401, 561

Goebbl U, 234

Goldblum RM, 252

Goldman AS, 252

Gomez NV, 396

Gomez RA, 396

Goodman SI, 311

Gorenenberg IAL, 379

Gourmelen M, 488

Gozal D, 204

Granoff DM, 16, 358

Greisen G, 445

Gruppuso PA, 599

Gunn AJ, 561

Haavisto A-M, 211

Haggerty R, S55

Hakanson DO, 571

Halkola LT, 383

Hamamoto K, 282

Hamaoka K, 483

Hamosh M, 32

Hargrave B, 140

Harrison HE, S62

Hashiro GM, 392

Hayashibe H, 472

Hehre D, 327

Heldt G, 137

Henderson DA, S56

Henderson WR, 466

Herschkowitz N, 566

Heubi JE, 26

Heymann M, 140

Hibbard JU, 80

Higgins RD, 580

Hintz RL, 45

Hirata $Y, 260$

Hochberg Z, 204

Hoch S, 109

Hoefler G, 304

Hoffmann RG, 148

Hollis BW, 26

Holzknecht RA, 408

Horstman SJ, 349

Howell DS, 41

Huang AS, 109

Huebers HA, 525

Huhtaniemi I, 21

Humme J, 52

Hyland K, 297

Ibata $Y, 483$

Igarashi T, 417

Ikeda K, 557

Ikegami M, 268, 592

Israels SJ, 337

Ito $\mathrm{S}, 11$

Ives NK, 436

Iwamoto HS, 282, 372

Jaffe M, 204
Janghorbani M, 36

Jansons RA, 181

Jobe A, 268

Jobe AH, 592

Johnston Jr RB, S44

Jordan SC, 353

Jori $\mathrm{G}, 22$

Julkunen MKA, 383

Kajio F, 557

Kamezawa Y, 11

Kamitsuka MD, 64

Kamoshita S, 417

Kampine JP, 148

Kang SS, 297

Kasahara Y, 227

Kato I, 11

Kato K, 227, 472

Kaufman FR, 117

Kleinman LI, 604

Klitzner TS, 144

Knip M, 353

Kobayashi Y, 260

Koenig JM, 583

Kojima T, 260

Kolanowski J, 161

Komeda T, 483

Kommareddy S, 612

Koren $G, 432$

Krugman S, S38

Kühl PG, 103

Kullama LK, 392

Kulovich S, 75

Kuribayashi T, 483

Lambert M, 297

Lang AB, 508

Le Bouc Y, 488

Lee PDK, 45

Leroux J-P, 497

Letarte J, 297

Leung DYM, 109

Levin SR, 353

Lewandoski JR, 574

Lewis K, 353

Liang X, 358

Lifschitz CH, 165

Lindner W, 278

Litov RE, 176

Lobo RA, 117

Loche S, 405

Loehr JP, 311

Lönnerdal B, 176

Lorentzon R, 41

Lorenz JM, 604

Lowenthal A, 297

Lowitt S, 293

Macdonald RA, 365

Madassery JV, 16

Malone JI, 293

Maloney JE, 122

Marescau B, 297

Maresh MJA, 622

Mariottini GI, 1

Markiewicz M, 588

Marple SL, 344, 349

Martinez AM, 52 
Mason GR, 70

Matsuzaki S, 260

Mauss D, 612

McKee KS, 525

McMillan EM, 337

Meeuwsen-Van der Roest WP, 256

Mehta NR, 32

Merritt AT, 137

Metlay LA, 460

Metsala TH, 383

Michelmann I, 234

Minczak BM, 547

Mink CM, 353

Miyawaki T, 227

Mizutani N, 297

Moawad AH, 80

Modanlou H, 612

Mohammed T, 622

Montaldo P, 1

Morville P, 140

Mosier HD, 181

Mughal MZ, 622

Mujsce DJ, 450

Müller EE, 405

Murdaugh E, 454

Nagaoka A, 483

Nahm MH, 16

Nakagawa M, 483

Nakahara T, 11

Nakamura KT, 392

Nakamura T, 483

Nakano S, 11

Nakazawa M, 557

Nathan DG, 423

Nelson SE, 36

Newburger JW, 109

$\mathrm{Ng} W G, 117$

Nichols BL, 525

Noma S, 11

Norris K, 612

Notter RH, 460

Novotny EJ, 566

Nürnberger W, 234

Nyhan WL, 75

Ogata ES, 56

Ogra PL, 153

Ohlsson L, 220

Oh W, 552

Okitsu S, 11

Oliver MH, 401

O'Shea JJ, 501

O'Toole C, 492

Oyedeji CO, 617

Packman S, 492

Padbury JF, 52

Palmer C, 332

Palmisano BW, 148

Parker KA, 588

Parrish MD, 476
Pastuszak A, 432

Pauly TH, 344, 349

Peach MJ, 396

Pearson HA, S4

Penney DP, 460

Perheentupa J, 215

Perry SF, 122

Pesonen E, 137

Petroff OAC, 566

Pettersson K, 211

Phelps DL, 580

Picciano MF, 32

Pintor C, 405

Pita JC, 41

Ponzoni M, 1

Possemiers I, 297

Prichard JW, 566

Pridjian G, 80

Prölss A, 256

Pryds O, 445

Quie PG, S1

Qureshi IA, 297

Raivio KO, 286

Raj JU, 70

Ramanathan E, 70

Raux-Demay M-C, 488

Ravin R, 144

Reddi E, 22

Redding GJ, 466

Reeds PJ, 165

Reinhart AL, 109

Rezvani I, 297

Riegel KP, 278

Riepenhoff-Talty M, 153

Riesenfeld T, 7

Rijkers GT, 514

Rinaldo P, 501

Roberton DM, 365

Robillard JE, 392

Robinson BH, 75

Rodrigues-Valverde V, 234

Roman C, 140

Roman CM, 282

Roncelli L, 234

Rosenberg AA, 454

Rosenberg LE, S10

Rosenfeld RG, 45

Rothman DL, 566

Rountree L, 45

Rubaltelli FF, 22

Rudolph AM, 282, 372

Russell GJ, 239

Ruz M, 170

Safar P, 264

Sahn DJ, 137

Said H, 519

Samuli Sarajas HS, 383

Santamore WP, 547

Saudubray J-M, 497

Schaad UB, 508
Schanler RJ, 252

Schneid H, 488

Schoeller DA, 98, 198

Scholte J, 497

Schonig G, 103

Schuette SA, 36

Schulman RG, 566

Schweer H, 103

Scott LA, 113

Scott MG, 16

Scriver CR, S17

Seagard JL, 148

Seger R, 234

Seidel M, 278

Seidler FJ, 181

Sennhauser FH, 365

Seurin D, 488

Seyberth HW, 103

Shackelford PG, 16

Shaffer TH, 547

Shapir Y, 144

Sharma A, 574

Shaw AJ, 622

Shelton MJ, 365

Shimoo K, 483

Shook LA, 349

Sibley CP, 622

Siimes ASI, 383

Silverman WA, S34

Simmons RA, 274

Skov LL, 445

Slotkin TA, 181

Smith AM, 32

Smits TM, 442

Snyderman SE, 297

Solomons NW, 170

Southall DP, 113

Stenman U-H, 215

Stezoski W, 264

Stijnen T, 379

Stonestreet BS, 552

Strang LB, 588

Sturman JA, 85

Suguihara C, 327

Swanson SL, 56

Sweetman L, 75

Synek B, 561

Szeto HH, 289

Tabor B, 268

Tai H-H, 349

Tajima M, 11

Takano N, 227

Takao A, 557

Tanaka K, 501

Taniguchi N, 227

Taniwaki H, 483

Tapanainen P, 215

Tedesco F, 234

Teitel DF, 372

Terheggen HG, 297

Thaler MM, 492

Thio SL, 52

Thompson GN, 413
Thompson R, 181

Thornhill B, 396

Tikkanen I, 137

Tonini GP, 1

Towfighi J, 332

Toyoda M, 353

Trachtman H, 85

Truog WE, 466

Tsang RC, 26

Tuchman M, 408

Uhari M, 353

Uhnoo IS, 153

Ushijima $\mathrm{H}, 11$

Välimäki IAT, 383

van de Ven C, 612

Van Hoof F, 161

Vannelli S, 405

Vannucci RC, 317, 332, 450

Vassilopoulou-Sellin R, 617

Verhoef J, 514

Versmold H, 256

Versmold HT, 278

Vettenranta K, 286

Villanueva D, 64

Vista N, 405

Volpe JJ, 186, S28

Vreeland PN, 64

Waggener TB, 113

Wahn V, 234

Wallen LD, 122

Walsh MM, 109

Walters DV, 588

Watkins PA, 304

Wedgwood J, 508

Welch RD, 501

Whitington PF, 80

Widness JA, 552

Williams CE, 561

Wilson DM, 45

Winter HS, 239

Wladimiroff JW, 379

Wolfson MR, 547

Wolin MJ, 165

Xu Y-K, 117

Yachie A, 227

Yamada T, 268, 592

Yokoi T, 227

Yoneda K, 344

Yoshida I, 75

Yoshino K, 11

Yoshino M, 297

Yu J, 181

Ziegler EE, 36

Zijlstra WG, 256, 442

Zimmerman JJ, 574

Zimran A, 234 